\title{
From Normal to Fast Walking: Impact of Cadence and Stride Length on Lower Extremity Joint Moments
}

\author{
Marzieh M Ardestani ${ }^{1 *}$, Christopher Ferrigno ${ }^{2,4}$, Mehran Moazen ${ }^{3}$, Markus A Wimmer ${ }^{4}$ \\ ${ }^{1}$ Department of Human Physiology, University of Oregon, Eugene, USA \\ ${ }^{2}$ Department of Anatomy and Cell Biology, Rush University Medical Center, Chicago, IL, USA \\ ${ }^{3}$ Department of Mechanical Engineering, University College London, Torrington Place, London WC1E \\ $7 \mathrm{JE}, \mathrm{UK}$ \\ ${ }^{4}$ Department of Orthopedic Surgery, Rush University Medical Center, Chicago, IL, USA
}

Work performed at Rush University Medical Center

\footnotetext{
Marzieh M Ardestani, PhD

Post-doctoral Research Fellow

Department of Human Physiology

University of Oregon

Oregon, 97403

Email: marziehm@uoregon.edu
} 


\begin{abstract}
This study aimed to clarify the influence of various speeding strategies (i.e. adjustments of cadence and stride length) on external joint moments. This study investigated the gait of 52 healthy subjects who performed self-selected normal and fast speed walking trials in a motion analysis laboratory. Subjects were classified into three separate groups based on how they increased their speed from normal to fast walking: (i) subjects who increased their cadence, (ii) subjects who increased their stride length and (iii) subjects who simultaneously increased both stride length and cadence. Joint moments were calculated using inverse dynamics and then compared between normal and fast speed trials within and between three groups using spatial parameter mapping.

Individuals who increased cadence, but not stride length, to walk faster did not experience a significant increase in the lower limb joint moments. Conversely, subjects who increased their stride length or both stride length and cadence, experienced a significant increase in all joint moments. Additionally, our findings revealed that increasing the stride length had a higher impact on joint moments in the sagittal plane than those in the frontal plane. However, both sagittal and frontal plane moments were still more responsive to the gait speed change than transverse plane moments. This study suggests that the role of speed in altering the joint moment patterns depends on the individual's speed-regulating strategy, i.e. an increase in cadence or stride length. Since the confounding effect of walking speed is a major consideration in human gait research, future studies may investigate whether stride length is the confounding variable of interest.
\end{abstract}

Keywords: Gait, Speed, Cadence, Stride Length, External Joint Moments 


\section{Introduction}

The existing body of literature is rich with studies analyzing how walking speed, an important consideration in gait analysis, may impact human gait biomechanics [1-6]. Significant associations have been found between gait speed and joint kinematics [7-10], joint kinetics [11, 12], muscle activities [13-15] and gait stability [16-19] in both healthy people and subjects with pathology. Cadence (number of steps per minute) and stride length are the key determinants of gait speed which describe important spatio-temporal aspects of human gait pattern [20]. The cadence and stride length relationship has been investigated as an indicator of gait control [21], gait abnormalities and fall risk [22]. Healthy subjects can regulate their gait speed by singularly adjusting the cadence, stride length or a combination of both strategies[23], with each strategy imparting excessive demands on different kinematics [24, 25]. Since the gait kinetic pattern is directly dictated through the interaction of joint kinematics [26], it is likely that different speeding strategies impact gait differently. To the best of our knowledge, a systematic evaluation of different speeding strategies (i.e. adjustments of cadence and stride length) and their role in altering the lower extremity joint moments has not been investigated in the literature. In addition, the speed-mediated effects have been studied only for discrete features of gait patterns such as "magnitudes" of joint moments defined at specific time points. In fact, available statistical analyses, such as t-test or analysis of variance (ANOVA) can only provide scalar-based hypotheses testing. Hence, many studies ignore the temporal information and dynamic patterns of gait by summarizing the complex waveforms with only a few scalars, and consequently fail to provide a more holistic understanding of the speed-mediated effects throughout the entire gait cycle.

This study used Spatial Parameter Mapping (SPM) to evaluate the waveforms for each gait parameter. SPM is a well-established technique for image comparison [27] that has recently been utilized in the field of biomechanics enabling the comparison of measurements at a vector level and over the entire gait waveform [28-32].The primary aim of this study was to understand how changes in cadence and stride length affect the external joint moments once a person switches from normal to fast walking speed. As a secondary aim, it was investigated which moments in what plane were affected the most. While this study does not directly aim to address the debate on the best technique for alleviating the confounding effects of speed in gait tests, it may provide additional insights on this matter. 


\section{Materials and Methods}

\subsection{Subjects}

Gait data from a total of 55 healthy asymptomatic adults were obtained from an IRB approved data repository at Rush University Medical Center. Since age has been shown to influence gait speed [29], subjects were chosen from a certain age ranging from 40 to 60 years. This age range is of interest in gait studies on lower extremity joint pathologies and was chosen to facilitate future comparison of the results. Inclusion criteria were: no clinical and structural (K/L grade $<2)$ evidence of complications such as osteoarthritis or rheumatoid arthritis in ankle, knee and hip joints, no significant lower limb pain over that past 2 months and no recent history of fracture or surgery in the lower limbs. Subjects needed to be pain-free at the day of testing with $<10 \mathrm{~mm}$ on the visual analog $100 \mathrm{~mm}$ score of the Western Ontario and McMaster Universities Arthritis Index (WOMAC)[33]. Since the external joint moments and other variables of interest had to be reprocessed, exclusion criteria included difficulties in data processing.

\subsection{Three dimensional gait analysis}

All tests were conducted between 2007 and 2008 by the same trained clinician and technical staff of the laboratory while an identical marker set and similar experimental condition were applied to all subjects. A total of six passive retroreflective markers were placed on the most lateral point of the superior iliac crest, the aspect of the greater trochanter, the lateral knee joint line, the lateral malleolus, the lateral most point on the calcaneus, and the head of the fifth metatarsal of the dominant limb. Markers were tracked with a sampling rate of $120 \mathrm{~Hz}$ using a gait analysis software system (CFTC - Computerized Functional Testing Corporation, Chicago, IL) with four optoelectronic cameras (Qualisys, Gothenburg, Sweden) [34]. Ground reaction forces (GRF) were measured using a multicomponent force plate (Bertec, Columbus, OHsampling rate of $120 \mathrm{~Hz}$ ). Each subject completed a total of six barefoot walking trials including three trials at self-selected normal speed and three trials at self-selected fast speed. Walking trials were deemed successful when the subject had a clean force plate strike on the indexed limb (the dominant leg). For every subject, the self-selected fast walking speed with an increase of at least one standard deviation higher than normal was accepted as self-selected fast speed. This criterion yielded a $>10 \%$ increase in the subjects' normal walking speed. Three-dimensional hip, knee and ankle joint moments were calculated using CFTC software. The software was based on a rigid link model of the lower extremities with no instantaneous rotation about the long axis of each segment and basic inverse dynamics. Details of the model have been described earlier[35]. External joint 
moments were then normalized to bodyweight times height (\%BW*HT) [36] and averaged over normal and fast walking trials for each subject. Sample frequency was normalized to 100 samples per gait cycle from $0 \%$ (heel strike) to $100 \%$ (the following heel strike of the same leg). Using a custom MATLAB script (version 2009, The Mathworks, Natick, MA, USA), the spatiotemporal variables (i.e. speed, stride length and cadence) were extracted from force plate measurements.

\subsection{Subject Clustering}

For each subject, the average of cadence $(\overline{\text { Cadence }})$ and stride length ( $\overline{\text { Stride }}$ ) were computed over the normal and fast walking trials. For each subject, the increments of cadence ( $\Delta$ Cadence ) and stride ( $\Delta$ Stride ) were then defined as follows:

$$
\begin{aligned}
& \Delta \text { Cadence }_{i}=\frac{\overline{\text { Cadence }_{i}^{\text {fast }}}-\overline{\text { Cadence }_{i}^{\text {normal }}}}{\overline{\text { Cadence }_{i}^{\text {normal }}}} \times 100 \% \quad i=\text { number of subject } \\
& \Delta \text { Stride }_{i}=\frac{\overline{\text { Stride }_{i}^{\text {fast }}}-\overline{\text { Stride }_{i}^{\text {normal }}}}{\overline{\text { Stride }_{i}^{\text {normal }}}} \times 100 \%
\end{aligned}
$$

Comparing the $\Delta$ Cadence and $\Delta$ Stride for each subject, participants were classified into one of the following groups:

I. Subjects who mainly increased their cadence to execute fast speed trials as $\left(\frac{\Delta \text { Cadence }}{\Delta \text { Stride }}\right)>1$.

II. Subjects who mainly increased their stride length to execute fast speed trials as $\left(\frac{\Delta \text { Cadence }}{\Delta \text { Stride }}\right)<1$

III. Subjects who increased both cadence and stride length to the same extent to increase their gait speed as $\left(\frac{\Delta \text { Cadence }}{\Delta \text { Stride }}\right) \cong 1$

\subsection{Statistical analysis}

One-way ANOVA with a significance level of $\mathrm{p}=0.05$ was used to compare subjects in terms of age, height, speed cadence and stride length. Spatial parameter mapping (SPM) was used to compare the waveforms of the external joint moments. SPM is a vector field analog for traditional statistical analyses such as t-test or ANOVA which provides a framework for continueslevel statistical comparison of biomechanical waveforms [28]. For a detailed description of SPM, see Pataky et al, 2010. In brief, for each vector (i.e. joint moment) a critical threshold was determined based on the smoothness and temporal increment of that vector over the gait cycle [32]. 
For each joint moment, t-statistics or F-statistics were calculated as a $(100 \times 1)$ continuum, referred as $\operatorname{SPM}(\mathrm{t})$ or $\operatorname{SPM}(\mathrm{F})$. If SPM exceeded the threshold, a significant difference is recognized. Using Random Field Theory [37], the probability with which this difference has occurred by chance was then calculated ( $\mathrm{p}$-value). Presented in this study, the vector-field equivalent of a paired t-test, $\operatorname{SPM}(\mathrm{t})$, was implemented to compare the joint moments between normal and fast speeds within each group, while the vector-field equivalent of a one-way ANOVA, SPM(F), was used to compare joint moments between groups at normal and fast speed trials. All of the aforementioned computations were conducted using "SPM1D", a free and open source software package for SPM, written in MATLAB software code [29].

\section{Results}

Three subjects were excluded due to difficulties in data processing, leaving 13 males and 39 females for inclusion with an average age of $54.2 \pm 6.0$ (years), body weight of $74.2 \pm 11.3(\mathrm{~kg})$ and a height of $1.60 \pm 0.07(\mathrm{~m})$. Overall, subjects increased their stride length and cadence at least one standard deviation higher than the corresponding values at normal speed walking condition. Subjects were divided into three groups based on the classification criteria described in section 2.3: 17 participants met group I criteria (increased cadence; $\frac{\Delta \text { Cadence }}{\Delta \text { Stride }}$ varied between 1.55 to 19.20), 14 participants met group II criteria (increased stride length; $\frac{\Delta \text { Cadence }}{\Delta \text { Stride }}$ varied between 0.025 to 0.75), and 21 participants met group III criteria (increased both cadence and stride length; with $\frac{\Delta \text { Cadence }}{\Delta \text { Stride }}$ varied between 0.93 to 1.17$)$. The demographic characteristics and the mean and standard deviation values of $\frac{\Delta \text { Cadence }}{\Delta \text { Stride }}$ for each group are described in Table 1 . There were no significant differences between these three groups in terms of age, height, weight and their speed, cadence and stride length at normal walking $(\mathrm{p}>0.05)$.

\subsection{Between group comparisons at normal speed:}

Using the SPM-equivalent of one-way ANOVA, joint moments were compared between the aforementioned groups over the whole gait cycle at normal speed (Appendix-Figure A.1). No significant differences were observed between hip, knee and ankle joint moments in frontal, sagittal or transverse planes. This comparison, in turn, reassured the homogeneity of the groups with regards to joint moments and built confidence that potential differences between these three groups at fast speed were related to speed adjustment strategies. 


\subsection{Within group comparison at fast speed}

The SPM-equivalent of a paired t-test was conducted to compare normal-speed and fastspeed joint moments within each group. Figure 1 shows the means and standard deviations of hip, knee and ankle joint moments, as well as the t-statistic values over one complete gait cycle. The shaded regions indicate portions of the gait cycle in which the t-statistic continuum passed the threshold (horizontal dashed lines). These areas indicate statistically-significant differences. Subjects who mainly increased their cadence to walk faster (group I) did not experience significant changes in their lower limb joint moments, and their corresponding SPM (t) values mostly remained between thresholds (see Figure 1). However, hip flexion $(\mathrm{p}=0.023)$ and hip adduction moments $(\mathrm{p}=0.016)$ increased during early stance, or $0-20 \%$ of the gait cycle[38]. Conversely, those subjects who mainly increased their stride length during fast speed gait (group II), experienced a significant increase in their hip, knee and ankle frontal and sagittal plane moments during early stance accompanied with a significant increase at 3D hip joint moments, and knee adduction moment during swing phase (60-80\% of gait cycle) whilst no significant increases were observed in joint moments either during the midstance or terminal stance phases (Figure 2). Similarly, subjects who simultaneously increased both cadence and stride length to increase gait speed (group III) experienced a significant increase in all joint moment components with the exception of their ankle adduction moment during early stance (Figure 3). Hip extension moment, hip internal rotation moment, knee flexion moment, knee rotation moment, ankle dorsi-flexion and ankle inversion moments were also increased significantly in group III during terminal stance (40\%-60\% gait cycle). During swing phase, hip flexion moment (at $80 \%$ to $95 \%$ of gait cycle) and hip rotation moment (at $60 \%-80 \%$ of gait cycle) were also significantly increased. It is noteworthy that hip and knee frontal plane moments increased solely at the first peak while ankle frontal moment increased at the second peak.

\subsection{Between group comparisons at fast speed}

Speed, cadence and stride length values were compared among three groups using oneway ANOVA test with significance level of $\mathrm{p}=0.05$. No significant group differences were observed in speed whilst stride length $(\mathrm{p}=0.014)$ and cadence $(\mathrm{p}=0.035)$ values were significantly different among three groups. The SPM equivalent of one-way ANOVA with Bonferroni-adjusted post hoc comparison was conducted to compare joint moments between three groups at fast walking speed. A comparison of hip joint moments is presented in Figure 4 as an example. For knee and ankle joint moments, refer to Appendix (Figures A.2 and A.3). As expected, group III demonstrated 
the largest increase in their joint moments during faster walking speeds. Comparing group I and II, subjects in group II experienced greater increases in their sagittal and frontal moments of hip, knee and ankle joints, while speed-mediated changes in the transverse moments were fairly similar between these two groups.

\section{Discussion}

The main purpose of this study was to investigate the effects of cadence and stride length on lower extremity joint moments when subjects increased their walking speed from normal to fast. Results suggest that, compared to the external joint moments elicited when subjects walked at their normal walking speeds, increasing cadence to walk faster had little effect on external joint moment patterns while increasing the stride length to walk faster led to remarkable increases in lower extremity joint moments .

SPM analysis is an enhanced statistical tool, which analyzes the dynamic changes of the waveforms, to obtain a more holistic understanding of joint moments between normal and fast speed walking conditions. Traditional statistical analyses, such as t-test or analysis of variance ANOVA, are common techniques to compare gait measurements between different walking conditions. Such basic analyses require that the dynamic waveforms be summarized with scalars. These discrete-time points (e.g. magnitudes of joint moments, or joint forces at maximum knee flexion angle) do not fully appreciate the underlying dynamics of gait waveforms.

Overall, the finding from this study that increasing the walking speed caused an increase in the peaks of hip and knee joint moments is consistent with the available literature: Shwartz et al (2008), Stansfield et al (2006) and Van Hamme et al (2015) conducted a linear regression analysis between peak values of joint moments and walking speed for children of different age range and demonstrated positive correlation between walking speed and joint moments with a greater influence on peaks than times of peak occurrence[6, 39, 40]. For adult population, several studies, assessing the relationship between gait speed and joint moments, also reported high relationship between peaks of joint moments in sagittal plane and walking speed [8, 41, 42]. On the other hand, the main focus of the present study was to indicate that speed may not be the right value to consider, but rather stride length is the key variable when looking at variations in moments. This was consistent with the study from Allet et al (2011) which showed that lower extremity joint moments significantly increase with an increase in the stride length [43]. SPM analysis, with a more detailed, time-based description of speed-mediated changes in the joint moments, revealed an overall increase in the hip flexion moment during $0-20 \%$ of the gait cycle, an increase in the hip extension 
and hip internal rotation moments during $50-70 \%$ of the gait cycle and a significant increase in the hip adduction and hip external rotation moments during 10-20\% gait cycle. This study analyzed a fairly large group of healthy, mid-aged participants who conducted their normal and fast walking trials over ground, avoiding potential confounding effects of treadmill use [42, 44, 45].

There is a long-standing debate on how to handle the speed-mediated effects with respect to joint moments [43]. In the newer literature, various techniques have been suggested to model the influence of speed on joint moments such as considering the gait speed as a covariate in statistical tests[33, 46], using an instrumented treadmill for gait tests and/or asking the subjects to walk at pre-determined speeds [40]. Each of these techniques however, is imperfect and has shortcomings which prevent them from being globally accepted. For example, considering the speed as a covariate violates a primary assumption of covariate analysis, namely a covariate being unrelated to the main effect [47]. Moreover, controlling the speed by asking subjects to walk at predetermined gait velocities, different from their comfortable walking pace, may significantly alter their gait patterns [47] and hence fail to reflect their natural patterns.

Despite the importance of cadence and stride length in gait analysis, their role in altering the lower extremity joint moments, has not been previously studied. To our knowledge, this study is the first to document the influence of various adjustments of cadence and stride length in fast speed walking condition with respect to joint moments. Our findings have potential technical implications, especially for treating the speed confounding effects on gait measurements. Astephen (2012) suggested that the best solution is "no solution" and caution should be taken in interpreting the results either when there are speed discrepancies between subjects or when the effect of speed has been taken out from the data set. Although the present study is not designed to address the handling of speed, a potential solution to the speed conundrum may be provided with the use of SPM waveform analysis and by considering the components of speed changes, namely cadence and stride length. Our findings suggest that the extent to which speed may confound gait measurements might depend on the habitual strategy that the individual subject may use to modulate his/her walking speed. In addition, regardless of the speed-regulating strategy, an increase in walking speed most influenced joint moments in the sagittal plane. Walking speed was progressively less influential on moments in the frontal plane and the transverse plane (see Figures 2 to 4). Hence, controlling for speed in a gait study may depend on the parameters of interest and the technique that subjects adopt to regulate their speed. For instance, for a gait study in which transverse plane moments are of interest and subjects rely on higher cadence to increase their walking speed, speed might not have any confounding effects on the variables on interest. 
The results of this study may contain clinically important, although preliminary, suggestions. Manipulating the stride length, cadence, and speed relationship could be a step toward tailored gait retraining or conservative speed-regulating strategy for individuals with joint pathology. Increasing cadence, rather than stride length, would likely impose fewer alterations on lower limb biomechanics, particularly on the frontal plane knee moment, which is important in the progression of medial knee osteoarthritis[48]. Hence, walking faster, executed through higher cadence, could be considered as a conservative speed-regulating strategy for those with knee joint pathology. Such gait retraining strategies, based on subtle changes in stride length or cadence, could be easily practiced and learned using a treadmill.

Several limitations of this study should be discussed. First and foremost, this is a retrospective cohort study and as such not perfectly controlled and thus limited clinical evidence. However, in the future, different combinations of stride length and cadence could be examined in prospective clinical trial in order to determine the optimum strategy leading to noticeably decreasing joint moments. Also, the findings made here using a healthy cohort may not be translatable to cohorts with joint disease, since patients with various lower extremity pathologies demonstrate abnormal gait patterns different from their healthy counterparts [49]. Hence, the generalizability of the results of this study to other subject groups has yet to be determined.

In conclusion, this study on healthy subjects between 40 and 60 years suggests that the role of speed in altering the joint moment patterns, particularly those in the frontal and sagittal planes, depends on the individual's speed-regulating strategy, i.e. whether an increase in cadence or stride length is used. Since the confounding effect of walking speed is a major consideration in human gait research, future studies may investigate whether stride length, rather than speed, is the confounding variable of interest when examining changes in the joint moments.

\section{Acknowledgement}

Authors would like to appreciate the motion laboratory at Rush University Medical Center for providing the data for this study. Also we would like to appreciate Professor Todd Pataky for his guidance on conducting the spatial parameter mapping analysis.

\section{References}

1. Bishop, M., et al., The effect of velocity on the strategies used during gait termination. Gait \& Posture, 2004. 20(2): p. 134-139. 
2. Chung, M.-J. and M.-J.J. Wang, The change of gait parameters during walking at different percentage of preferred walking speed for healthy adults aged 20-60 years. Gait \& Posture, 2010. 31(1): p. 131-135.

3. Jordan, K., J.H. Challis, and K.M. Newell, Walking speed influences on gait cycle variability. Gait \& Posture, 2007. 26(1): p. 128-134.

4. Kang, H.G. and J.B. Dingwell, Separating the effects of age and walking speed on gait variability. Gait \& Posture, 2008. 27(4): p. 572-577.

5. Rosenbaum, D., M. Westhues, and K. Bosch, Effect of gait speed changes on foot loading characteristics in children. Gait \& Posture, 2013. 38(4): p. 1058-1060.

6. Schwartz, M.H., A. Rozumalski, and J.P. Trost, The effect of walking speed on the gait of typically developing children. Journal of Biomechanics, 2008. 41(8): p. 1639-1650.

7. Hanlon, M. and R. Anderson, Prediction methods to account for the effect of gait speed on lower limb angular kinematics. Gait \& Posture, 2006. 24(3): p. 280-287.

8. Lelas, J.L., et al., Predicting peak kinematic and kinetic parameters from gait speed. Gait \& Posture, 2003. 17(2): p. 106-112.

9. Røislien, J., et al., Simultaneous estimation of effects of gender, age and walking speed on kinematic gait data. Gait \& Posture, 2009. 30(4): p. 441-445.

10. Swinnen, E., et al., Walking more slowly than with normal velocity: The influence on trunk and pelvis kinematics in young and older healthy persons. Clinical Biomechanics, 2013. 28(7): p. 800-806.

11. Okita, Y., et al., The effect of walking speed on gait kinematics and kinetics after endoprosthetic knee replacement following bone tumor resection. Gait \& Posture, 2014. 40(4): p. 622-627.

12. Roemmich, R.T., E.L. Stegemöller, and C.J. Hass, Lower extremity sagittal joint moment production during split-belt treadmill walking. Journal of Biomechanics, 2012. 45(16): p. 2817-2821.

13. den Otter, A.R., et al., Speed related changes in muscle activity from normal to very slow walking speeds. Gait \& Posture, 2004. 19(3): p. 270-278.

14. Ranisavljev, I., et al., The relationship between hip, knee and ankle muscle mechanical characteristics and gait transition speed. Human Movement Science, 2014. 38(0): p. 4757.

15. Tirosh, O. and W.A. Sparrow, Age and walking speed effects on muscle recruitment in gait termination. Gait \& Posture, 2005. 21(3): p. 279-288.

16. Caderby, T., et al., Influence of gait speed on the control of mediolateral dynamic stability during gait initiation. Journal of Biomechanics, 2014. 47(2): p. 417-423.

17. Espy, D.D., et al., Independent influence of gait speed and step length on stability and fall risk. Gait \& Posture, 2010. 32(3): p. 378-382.

18. Hak, L., et al., Steps to Take to Enhance Gait Stability: The Effect of Stride Frequency, Stride Length, and Walking Speed on Local Dynamic Stability and Margins of Stability. PLoS ONE, 2013. 8(12): p. e82842.

19. Krasovsky, T., et al., Effects of walking speed on gait stability and interlimb coordination in younger and older adults. Gait \& Posture, 2014. 39(1): p. 378-385.

20. MURRAY, M.P., et al., COMPARISON OF FREE AND FAST SPEED WALKING PATTERNS OF NORMAL MEN. American Journal of Physical Medicine \& Rehabilitation, 1966. 45(1): p. 824.

21. Egerton, T., et al., Central gait control mechanisms and the stride length - cadence relationship. Gait \& Posture, 2011. 34(2): p. 178-182. 
22. Callisaya, M.L., et al., Risk of falls in older people during fast-walking - The TASCOG study. Gait \& Posture, 2012. 36(3): p. 510-515.

23. Howard, C., C. Wallace, and D.S. Stokic, Stride length-cadence relationship is disrupted in below-knee prosthesis users. Gait \& Posture, 2013. 38(4): p. 883-887.

24. Danion, F., et al., Stride variability in human gait: the effect of stride frequency and stride length. Gait \& Posture, 2003. 18(1): p. 69-77.

25. Huang, Y., et al., The effects of stride length and stride frequency on trunk coordination in human walking. Gait \& Posture, 2010. 31(4): p. 444-449.

26. Ardestani, M.M., M. Moazen, and Z. Jin, Sensitivity analysis of human lower extremity joint moments due to changes in joint kinematics. Medical Engineering \& Physics, 2015. 37(2): p. 165-174.

27. Friston, K.J., et al., Statistical parametric maps in functional imaging: a general linear approach. Human brain mapping, 1994. 2(4): p. 189-210.

28. Pataky, T.C., Generalized n-dimensional biomechanical field analysis using statistical parametric mapping. Journal of Biomechanics, 2010. 43(10): p. 1976-1982.

29. Pataky, T.C., One-dimensional statistical parametric mapping in Python. Computer Methods in Biomechanics and Biomedical Engineering, 2011. 15(3): p. 295-301.

30. Pataky, T.C., et al., New insights into the plantar pressure correlates of walking speed using pedobarographic statistical parametric mapping (pSPM). Journal of Biomechanics, 2008. 41(9): p. 1987-1994.

31. Pataky, T.C., M.A. Robinson, and J. Vanrenterghem, Vector field statistical analysis of kinematic and force trajectories. Journal of Biomechanics, 2013. 46(14): p. 2394-2401.

32. Robinson, M.A., J. Vanrenterghem, and T.C. Pataky, Statistical Parametric Mapping (SPM) for alpha-based statistical analyses of multi-muscle EMG time-series. Journal of Electromyography and Kinesiology, 2014. in press.

33. McConnell, S., P. Kolopack, and A.M. Davis, The Western Ontario and McMaster Universities Osteoarthritis Index (WOMAC): a review of its utility and measurement properties. Arthritis care \& research, 2001. 45(5): p. 453-461.

34. Hurwitz, D.E., et al., The knee adduction moment during gait in subjects with knee osteoarthritis is more closely correlated with static alignment than radiographic disease severity, toe out angle and pain. Journal of Orthopaedic Research, 2002. 20(1): p. 101107.

35. Hurwitz, D.E., et al., Hip motion and moments during gait relate directly to proximal femoral bone mineral density in patients with hip osteoarthritis. Journal of Biomechanics, 1998. 31(10): p. 919-925.

36. Moisio, K.C., et al., Normalization of joint moments during gait: a comparison of two techniques. Journal of Biomechanics, 2003. 36(4): p. 599-603.

37. Friston, K.J., et al., Statistical parametric maps in functional imaging: A general linear approach. Human Brain Mapping, 1994. 2(4): p. 189-210.

38. Perry, J. and J.R. Davids, Gait analysis: normal and pathological function. Journal of Pediatric Orthopaedics, 1992. 12(6): p. 815.

39. Stansfield, B., et al., Regression analysis of gait parameters with speed in normal children walking at self-selected speeds. Gait \& Posture, 2006. 23(3): p. 288-294.

40. Van Hamme, A., et al., Gait parameters database for young children: The influences of age and walking speed. Clinical Biomechanics, 2015.

41. Alcock, L., N. Vanicek, and T. O'Brien, Alterations in gait speed and age do not fully explain the changes in gait mechanics associated with healthy older women. Gait \& Posture, 2013. 37(4): p. 586-592. 
42. Watt, J.R., et al., A three-dimensional kinematic and kinetic comparison of overground and treadmill walking in healthy elderly subjects. Clinical Biomechanics, 2010. 25(5): p. 444449.

43. Allet, L., et al., The influence of stride-length on plantar foot-pressures and joint moments. Gait \& Posture, 2011. 34(3): p. 300-306.

44. Wearing, S.C., L.F. Reed, and S.R. Urry, Agreement between temporal and spatial gait parameters from an instrumented walkway and treadmill system at matched walking speed. Gait \& Posture, 2013. 38(3): p. 380-384.

45. Rozumalski, A., et al., Treadmill vs. overground running gait during childhood: $A$ qualitative and quantitative analysis. Gait \& Posture, 2015. 41(2): p. 613-618.

46. Lim, Y.P., Y.-C. Lin, and M.G. Pandy, Muscle function during gait is invariant to age when walking speed is controlled. Gait \& Posture, 2013. 38(2): p. 253-259.

47. Astephen Wilson, J.L., Challenges in dealing with walking speed in knee osteoarthritis gait analyses. Clinical Biomechanics, 2012. 27(3): p. 210-212.

48. Miyazaki, T., et al., Dynamic load at baseline can predict radiographic disease progression in medial compartment knee osteoarthritis. Annals of the Rheumatic Diseases, 2002. 61(7): p. 617-622.

49. Federolf, P.A., K.A. Boyer, and T.P. Andriacchi, Application of principal component analysis in clinical gait research: Identification of systematic differences between healthy and medial knee-osteoarthritic gait. Journal of Biomechanics, 2013. 46(13): p. 2173-2178. 

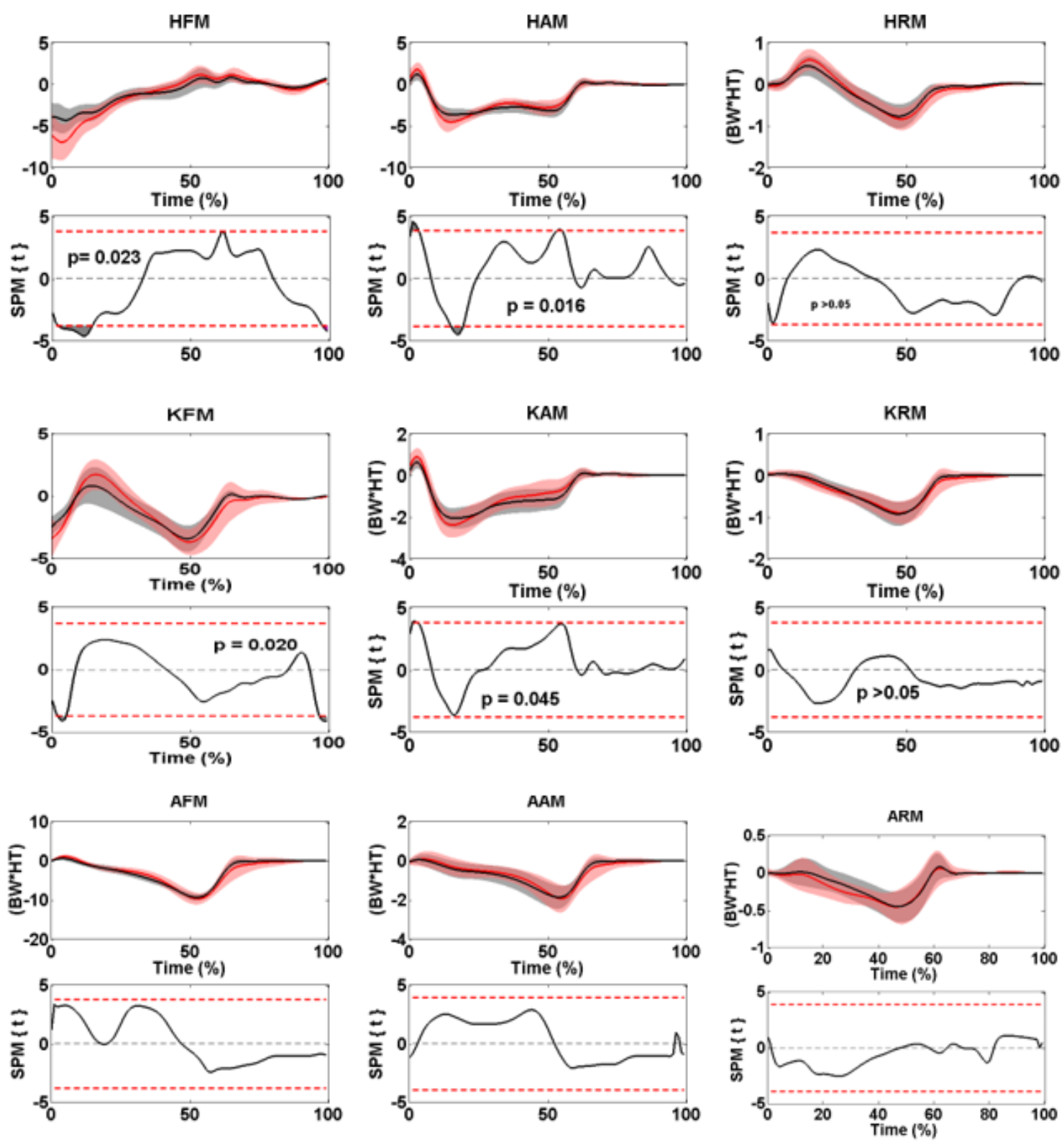

Figure 1 Comparison of average (solid line) and standard deviation (cloud) of (a) hip joint moments, (b) knee joint moments, and (c) ankle joint moments between normal (black) and fast(red) speed walking trials for subjects in group I. Abbreviations are t-statistic (SPM(t $\})$, hip flexion moment (HFM), hip adduction moment (HAM), hip rotation moment(HRM), knee flexion moment (KFM), knee adduction moment (KAM), knee rotation moment (KRM), ankle flexion moment(AFM), ankle adduction moment(AAM) and ankle rotation moment(ARM). The horizontal dotted line indicates the critical thresholds $\left(t^{*}\right)$. Regions of gait cycle for which SPM

$\{t\}$ exceeded the critical threshold, were considered as statistically significant differences. 

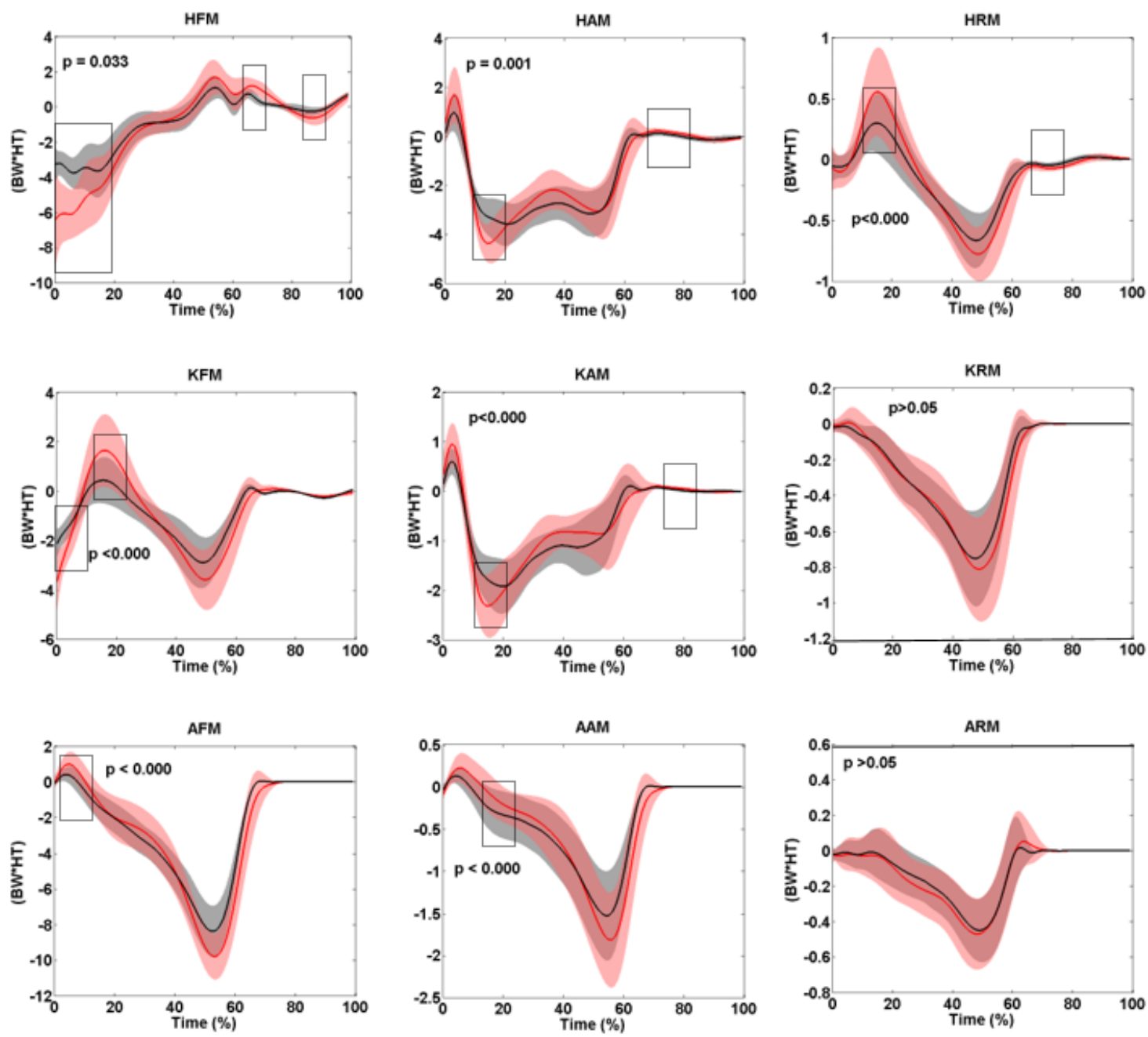

Figure 2 Comparison of average (solid line) and standard deviation (cloud) of (a) hip joint moments, (b) knee joint moments, and (c) ankle joint moments between normal (black) and fast(red) speed walking trials for subjects in group II. Abbreviations are hip flexion moment (HFM), hip adduction moment (HAM), hip rotation moment(HRM), knee flexion moment

(KFM), knee adduction moment (KAM),knee rotation moment (KRM), ankle flexion moment(AFM), ankle adduction moment(AAM) and ankle rotation moment(ARM). Regions of gait cycle for which SPM $\{\mathrm{t}\}$ exceeded the critical threshold (statistically significant differences) are specified with rectangular frames 

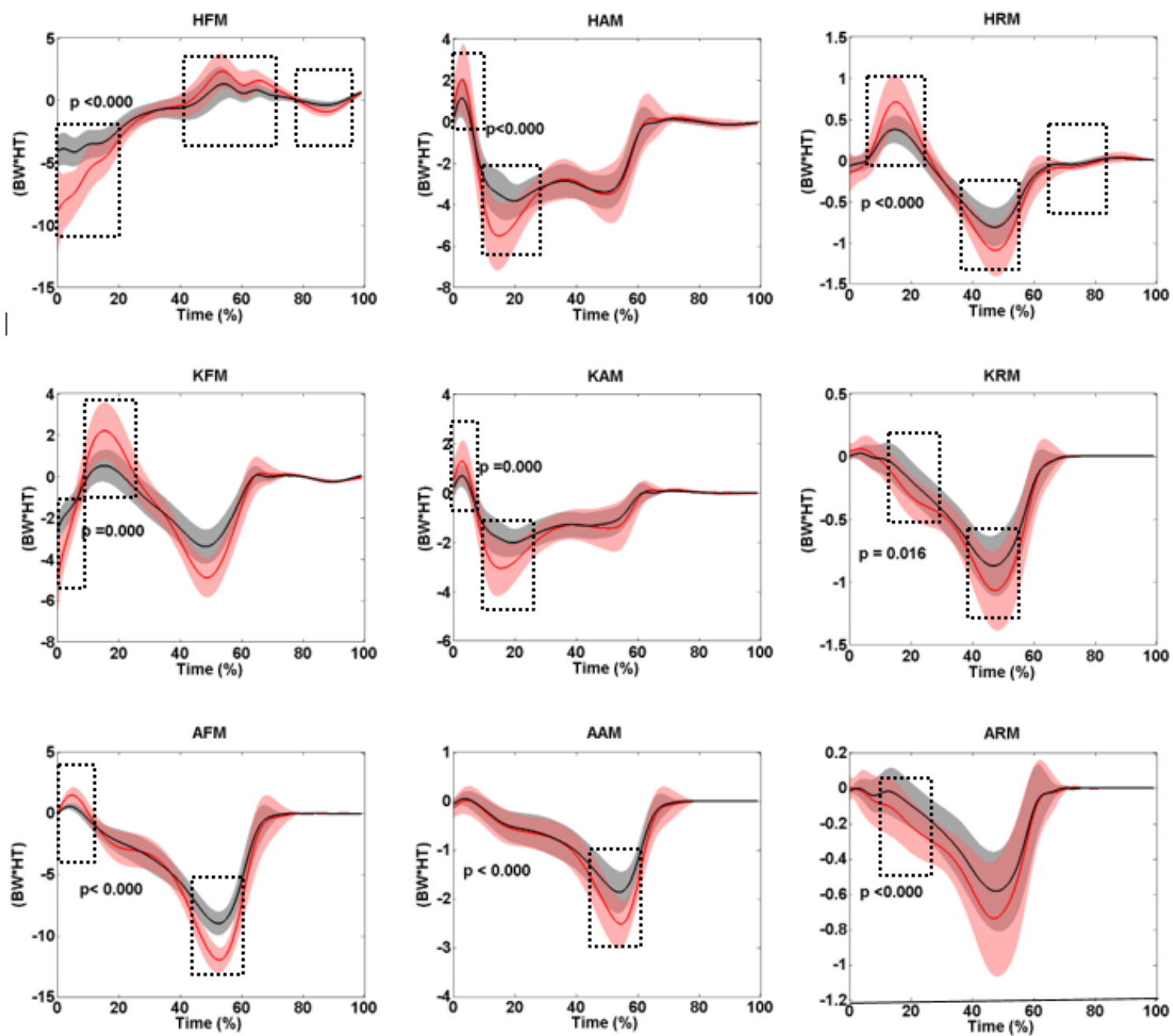

Figure 3 Comparison of average (solid line) and standard deviation (cloud) of (a) hip joint moments, (b) knee joint moments, and (c) ankle joint moments between normal (black) and fast(red) speed walking trials for subjects in group III. Abbreviations are hip flexion moment (HFM), hip adduction moment (HAM), hip rotation moment(HRM), knee flexion moment

(KFM), knee adduction moment (KAM),knee rotation moment (KRM), ankle flexion moment(AFM), ankle adduction moment(AAM) and ankle rotation moment(ARM). Regions of gait cycle for which SPM $\{\mathrm{t}\}$ exceeded the critical threshold (statistically significant differences) are specified with rectangular frames. 

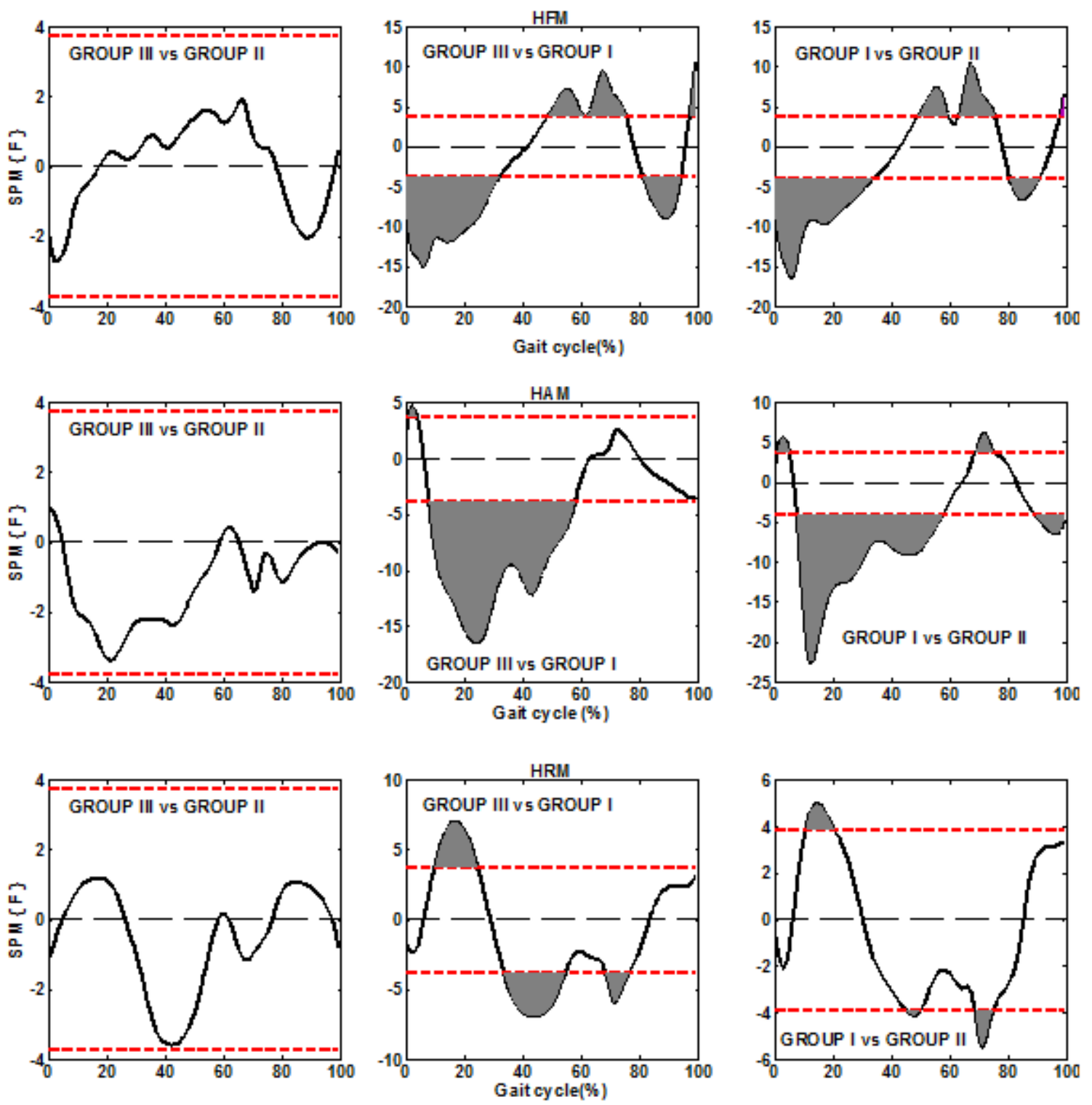

Figure 4 Comparison of hip flexion moment (HFM), hip adduction moment (HAM) and hip rotation moment (HRM) between three groups at fast speed. SPM $\{F\}$ represents F-statistic computation between groups. The horizontal dotted line indicates the critical thresholds $\left(\mathrm{F}^{*}\right)$. Regions of gait cycle for which SPM $\{F\}$ exceeded the critical threshold, were considered as statistically significant differences. 


\section{Appendix}
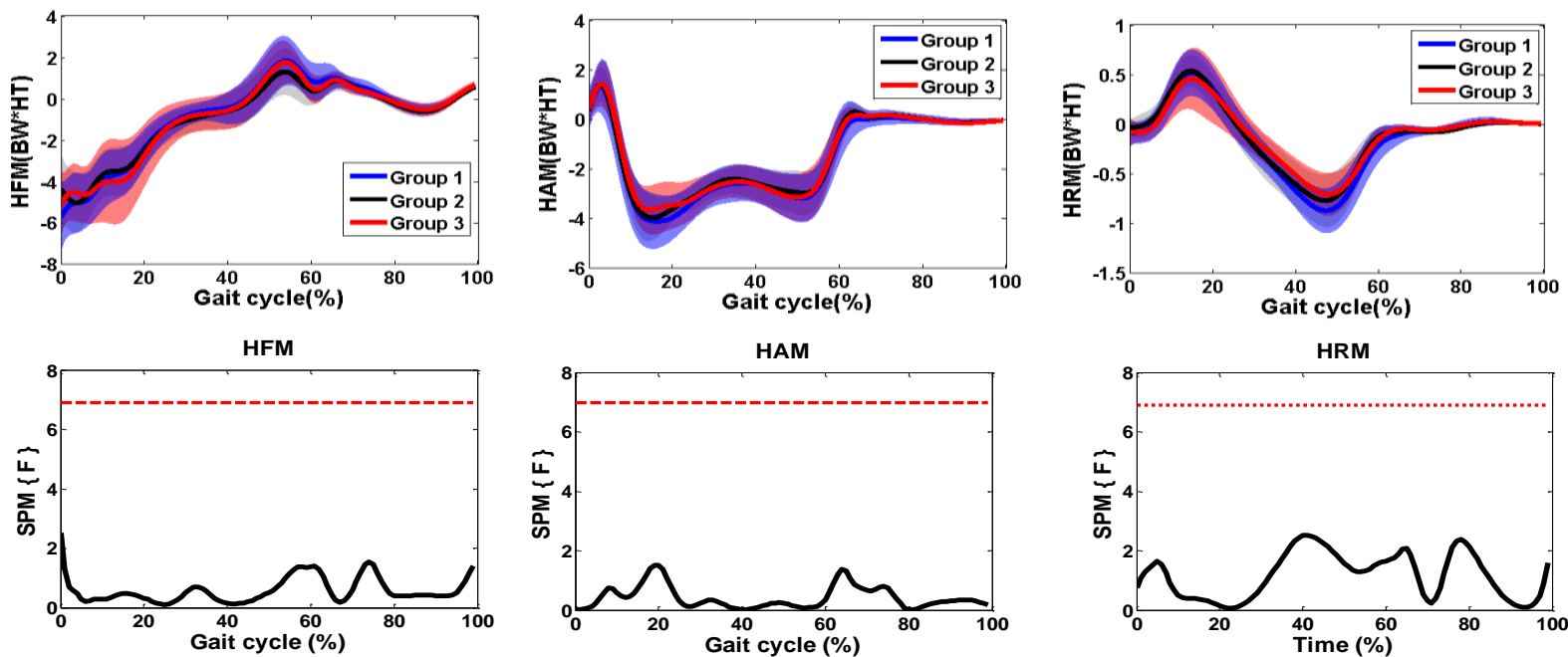

(a)
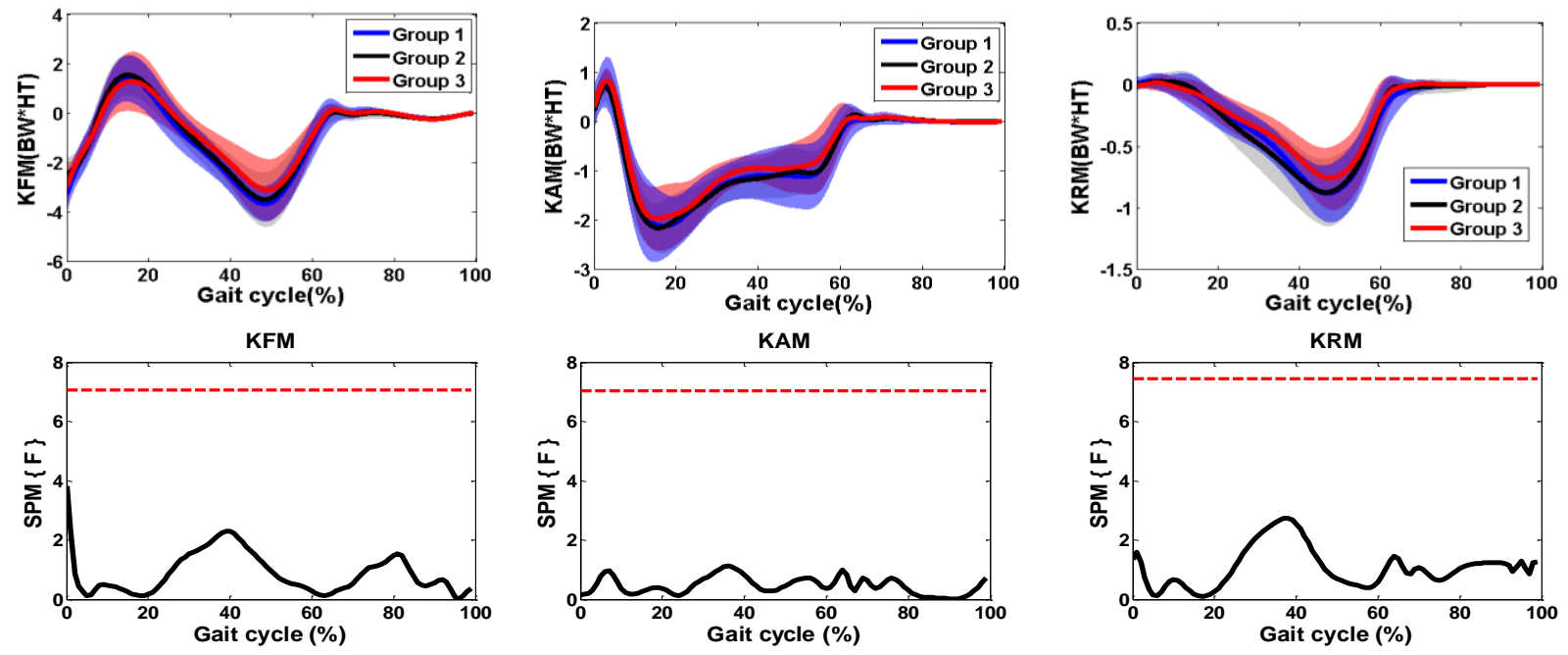

(b)
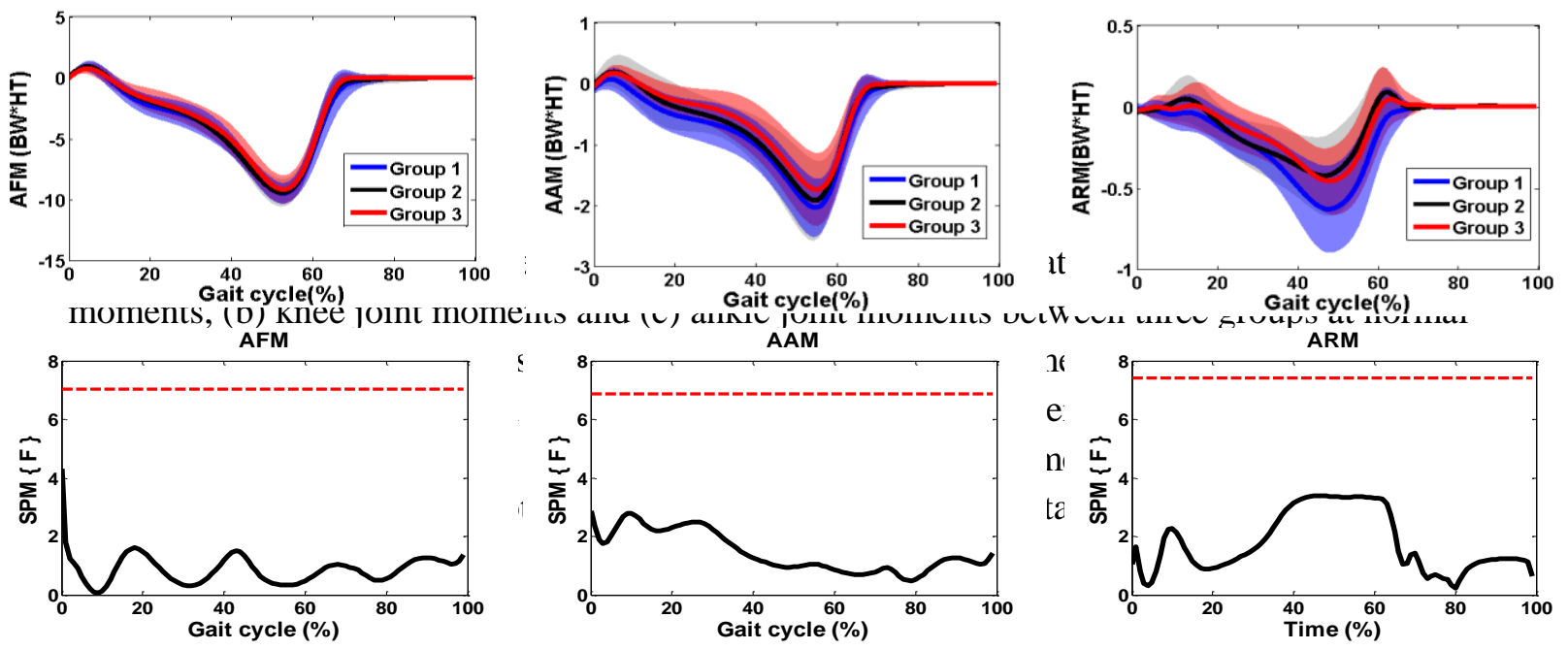
critical thresholds $\left(\mathrm{F}^{*}\right)$. Regions of gait cycle for which SPM $\{\mathrm{F}\}$ exceeded the critical threshold, were considered as statistically significant differences. 

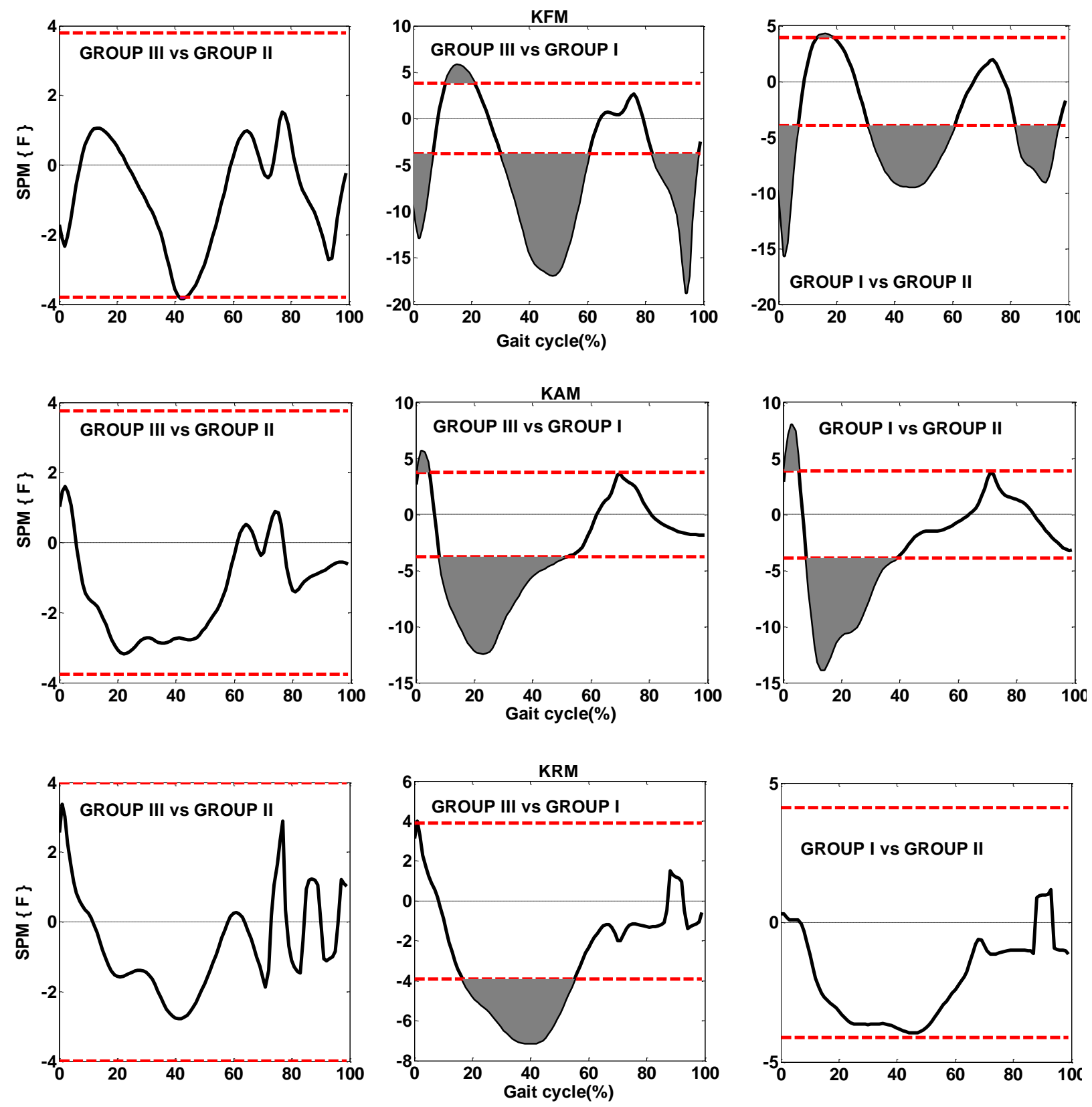

Figure A.2. Comparison of knee flexion moment (KFM), knee adduction moment (KAM) and knee rotation moment (KRM) between three groups at fast speed. SPM $\{F\}$ represents F-statistic computation between groups. The horizontal dotted line indicates the critical thresholds $\left(\mathrm{F}^{*}\right)$. Regions of gait cycle for which SPM $\{\mathrm{F}\}$ exceeded the critical threshold, were considered as statistically significant differences. 

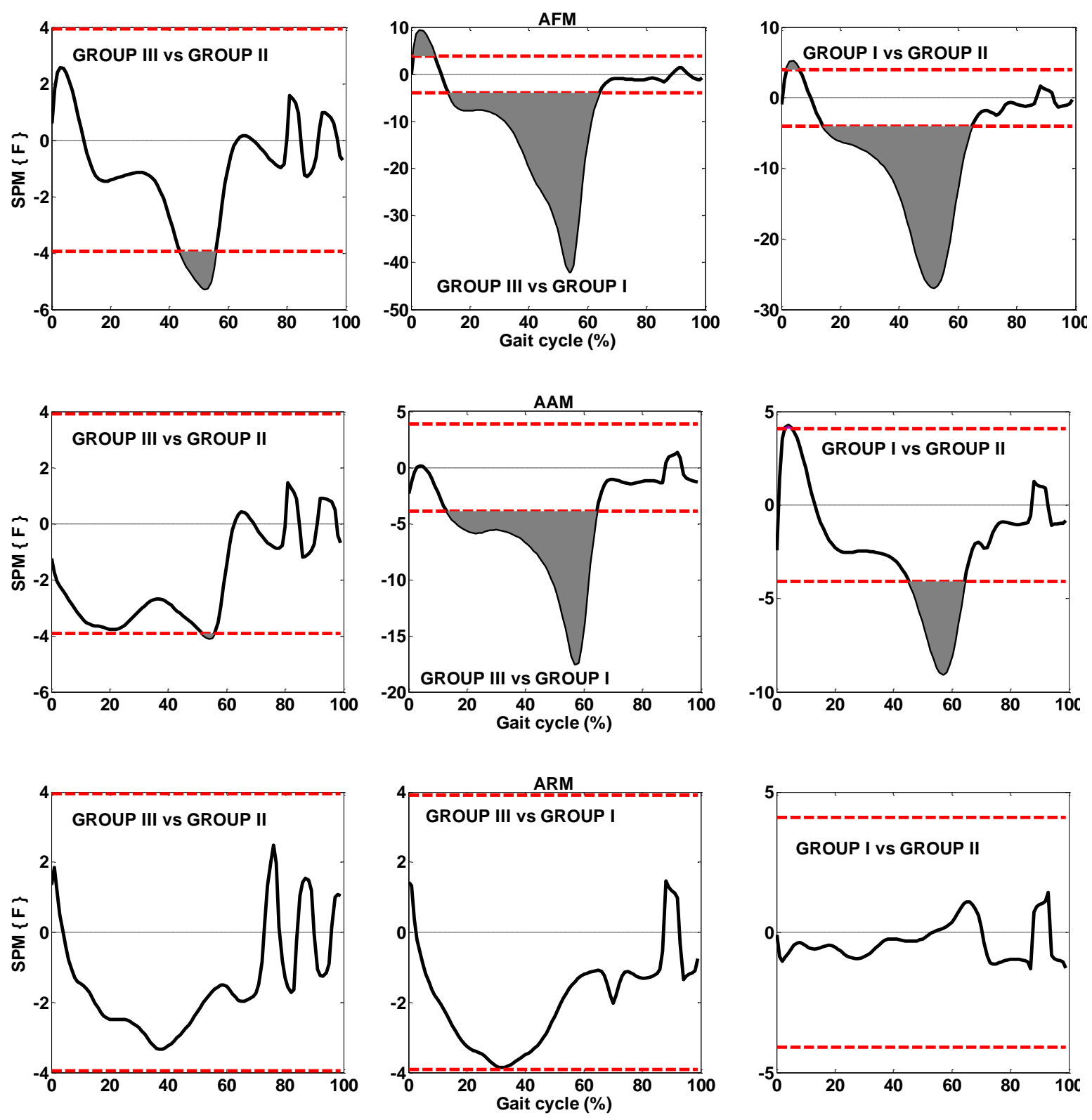

Figure A.3. Comparison of ankle flexion moment (AFM), ankle adduction moment (AAM) and ankle rotation moment (ARM) between three groups at fast speed. SPM $\{F\}$ represents F-statistic computation between groups. The horizontal dotted line indicates the critical thresholds $\left(\mathrm{F}^{*}\right)$. Regions of gait cycle for which SPM $\{\mathrm{F}\}$ exceeded the critical threshold, were considered as statistically significant differences. 
Table A.1 Demographic description of three groups (mean \pm standard deviation)

\begin{tabular}{|c|c|c|c|c|c|c|c|c|c|c|}
\hline \multirow[t]{2}{*}{ Group } & \multirow{2}{*}{$\begin{array}{l}\text { Age } \\
\text { (year) }\end{array}$} & \multirow{2}{*}{$\begin{array}{c}\text { Height } \\
\text { (m) }\end{array}$} & \multirow{2}{*}{$\begin{array}{c}\text { Weight } \\
\text { (N) }\end{array}$} & \multicolumn{3}{|c|}{ Normal walking } & \multicolumn{3}{|c|}{ Fast walking } & \multirow{2}{*}{$\frac{\Delta \text { Cadence }}{\Delta \text { Stride }}$} \\
\hline & & & & $\begin{array}{l}\text { Speed } \\
(\mathrm{m} / \mathrm{sec})\end{array}$ & $\begin{array}{l}\text { Stride } \\
\text { (m) }\end{array}$ & $\begin{array}{c}\text { Cadence } \\
\text { (\#steps/min) }\end{array}$ & $\begin{array}{l}\text { Speed } \\
\text { (m/sec) }\end{array}$ & $\begin{array}{l}\text { Stride } \\
\text { (m) }\end{array}$ & $\begin{array}{c}\text { Cadence } \\
\text { (\#steps/min) }\end{array}$ & \\
\hline $\begin{array}{l}\text { Group I } \\
(\mathrm{N}=17)\end{array}$ & $56.17 \pm 6.19$ & $1.65 \pm 0.07$ & $743.39 \pm 124.82$ & $1.35 \pm 0.25$ & $1.38 \pm 0.14$ & $116.78 \pm 13.72$ & $1.60 \pm 0.31$ & $1.44 \pm 0.14$ & $132.17 \pm 16.89$ & $4.24 \pm 4.66$ \\
\hline $\begin{array}{l}\text { Group II } \\
(\mathrm{N}=14)\end{array}$ & $52.57 \pm 6.18$ & $1.69 \pm 0.06$ & $741.31 \pm 115.64$ & $1.31 \pm 0.26$ & $1.39 \pm 0.17$ & $112.36 \pm 15.21$ & $1.56 \pm 0.24$ & $1.57 \pm 0.16$ & $119.06 \pm 13.22$ & $0.44 \pm 0.21$ \\
\hline $\begin{array}{l}\text { Group III } \\
(\mathrm{N}=21)\end{array}$ & $53.84 \pm 5.88$ & $1.63 \pm 0.07$ & $716.49 \pm 97.06$ & $1.27 \pm 0.16$ & $1.31 \pm 0.10$ & $115.93 \pm 9.76$ & $1.50 \pm 0.19$ & $1.42 \pm 0.12$ & $126.20 \pm 9.27$ & $1.03 \pm 0.11$ \\
\hline
\end{tabular}

